\title{
Diffusion and Reactions in Gold Films
}

\section{A REVIEW OF FUNDAMENTAL ASPECTS}

\author{
John M. Poate
}

Bell Laboratories, Murray Hill, N.J., U.S.A.

\author{
The processes of interdiffusion and chemical reaction in thin film \\ systems are of great scientific and technological interest, and our \\ understanding of them has been greatly helped by the study of gold \\ films. In this review the author deals with fundamental advances in this \\ field with particular reference to the systems of importance to the elec- \\ tronics industry.
}

Thin metal films occupy a position of crucial importance in electronic circuitry, where they serve extensively as conducting paths and in connectors. The prime requirement for such applications is that metallizations have an intrinsically high conductivity. Additionally they must be resistant to electromigration and to corrosion failure. At first sight, gold which possesses these properties to an exceptional degree would appear to be the ideal material, but aluminium is, in fact, the most widely used metallization in integrated circuits. One reason for this is clearly material cost. Another perhaps more important reason is the inability of gold, as opposed to aluminium, to adhere to oxidized silicon. This latter problem may be overcome by the introduction of intermediate 'bonding metals' such as titanium or chromium. The resulting bi-layer structures are, however, susceptible to corrosion, and a third "barrier' metallization of palladium or platinum has to be introduced between the gold and the adhesion layer. It is not surprising that designers of integrated circuits, confronted with a choice between such complex systems and metallization with a single layer of aluminium, have opted largely for the latter. Nevertheless, as described by Pinnel (1) and Holloway (2) in this journal, gold films are used extensively in circuitry where a high degree of reliability is required and where the added complexity of fabrication is not an overriding consideration.

In addition to their application in conductor paths, metal films are used in ohmic and Schottky contacts to semiconductors. Gold-based alloys are widely employed in ohmic contacts to compound semiconductors, the contact being formed by alloying the metallization directly with the underlying semiconductor. However, gold films are uncommon in ohmic contacts to silicon devices since gold and silicon react somewhat uncontrollably at metallurgically very low temperatures. In Schottky barriers on silicon, gold again finds wide application in the form of surface barrier detectors for charged particles. These devices have had considerable impact on nuclear spectroscopy and, as will be shown later, have thereby contributed significantly to the study of thin film reactions.

Clearly, the key to successful application of thin films is an understanding of the mechanisms of diffusion and reaction processes which occur in them. The characterization of reactions between films only a few hundred nanometres in thickness calls for the analysis of layers 10 to 1000 atoms in thickness located a similar distance beneath the surface. In the 1960's no experimental technique was available for doing this. It was therefore a happy circumstance that, as the importance of thin film reactions in technological applications became increasingly evident in the 1970's, such techniques became available.

\section{Experimental Techniques}

In the study of interdiffusion and compound formation in thin films, lateral uniformity of structure and composition is usually assumed. Such diffusion and reaction processes therefore result in compositional changes in a direction normal to the plane of the films and are particularly evident close to interfaces. The latter can display either sharply delineated boundaries, or layers of chemical compounds resulting from reaction between the adjacent species, or regions of interdiffusion (Figure 1). The essential determination of composition as a function of depth is at present achieved predominantly by the two techniques illustrated in Figure 2. The first (Figure 2(a)) employs beams of high energy particles, typically helium- 4 ions in the $\mathrm{MeV}$ range of energy, which during their 
Fig. 1 The interaction of two thin films, $A$ and $B$, on a substrate, showing uniform films with a well defined planar interface (top), reaction between $A$ and $B$ to form a compound having the stoichiometry $A_{x} B$ and defined interfaces (middle), and the interdiffusion of $A$ and $B$ with faster diffusion along grain boundaries thus forming a poorly defined interface at the (bottom)

inward and/or outward passage through the sample lose energy in a manner which is characteristic of the nature and concentrations of the species present. In the most widely employed version of this particle energy loss technique, Rutherford backscattering spectroscopy (RBS), the backscattered ions are analyzed by the gold/silicon surface barrrier detectors which were mentioned earlier. The second technique (Figure 2(b)) involves progressive erosion of the sample by sputtering with a beam of particles, typically argon ions in the $\mathrm{keV}$ range of energy, and the analysis of the newly exposed surface layers by Auger electron spectroscopy (AES) or by detection of the sputtered species.

Early investigators of thin film behaviour had no recourse to such powerful analytical techniques. The first published work on interdiffusion in thin films, that of DuMond and Youtz (3) in 1935, pointed out the applicability of X-ray diffraction to the study of low-temperature diffusion in alternating layers of copper and gold. The authors stated: 'It would seem likely that we have here an excellent way of studying intimately the diffusion of atoms in the solid state.' In later work (4) they succeeded in determining the diffusion coefficient of gold in copper at room temperature. Although such indirect methods of determining interdiffusion coefficients remained useful in the intervening years, the most active research groups, with few exceptions, now employ either the particle energy loss or the sputtersectioning technique. The recent emergence of RBS, in particular, has marked a significant advance in the field of thin film analysis. Techniques such as optical microscopy and scanning electron microscopy naturally remain indispensable in the determination

Fig. 2 Two methods of obtaining concentration against depth profiles in thin films. (a) The particle energy loss techniques use collimated incident beams of accelerated ions which lose energy by interaction with electrons or by nuclear scattering during their inward and outward passage through the film. The energy of the backscattered ions is generally analyzed using surface barrier semiconductor detectors. (b) The sputter or erosion sectioning techniques rely on the exposure of progressively deeper layers of film which can be analyzed either by Auger electron spectroscopy or by detection of the sputtered species
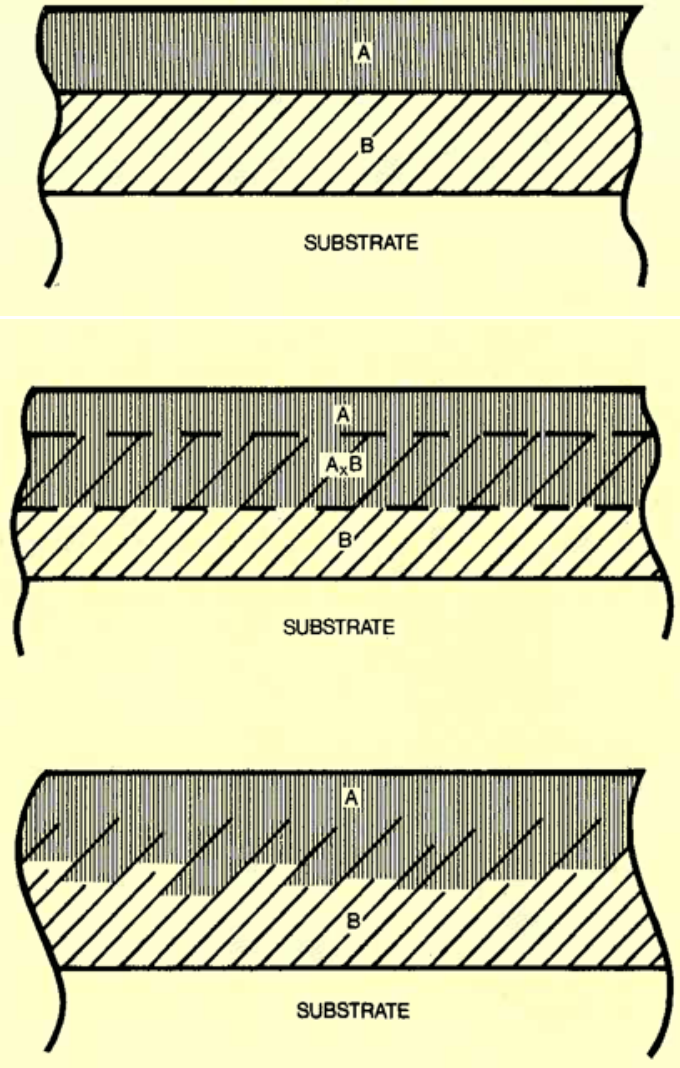

(a) PARTICLE ENERGY LOSS

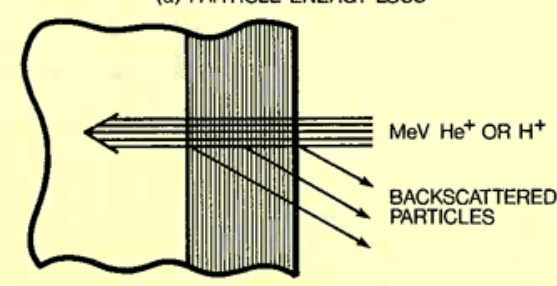

(b) SPUTTER SECTIONING

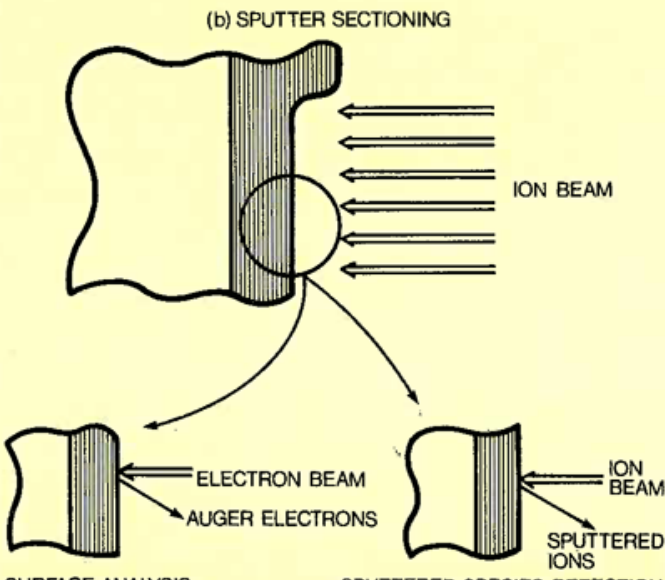

SURFACE ANALYSIS
SPUTTERED SPECIES DETECTION 
of the lateral structures of films. Electron and X-ray diffraction measurements are equally essential to the identification of thin film reaction products.

\section{Fundamental Considerations}

For an understanding of the processes and reactions which occur in thin film systems, a consideration of the equilibrium phase diagrams of the alloy systems which are involved is essential. The binary phase diagrams for the gold-silver, gold-silicon and goldaluminium systems (see Figure 3) are three important examples. These demonstrate the progression from a complete series of solid solutions to a simple eutectic and, finally, to a range of intermetallic compounds. Certain features of thin film reactions can be in-
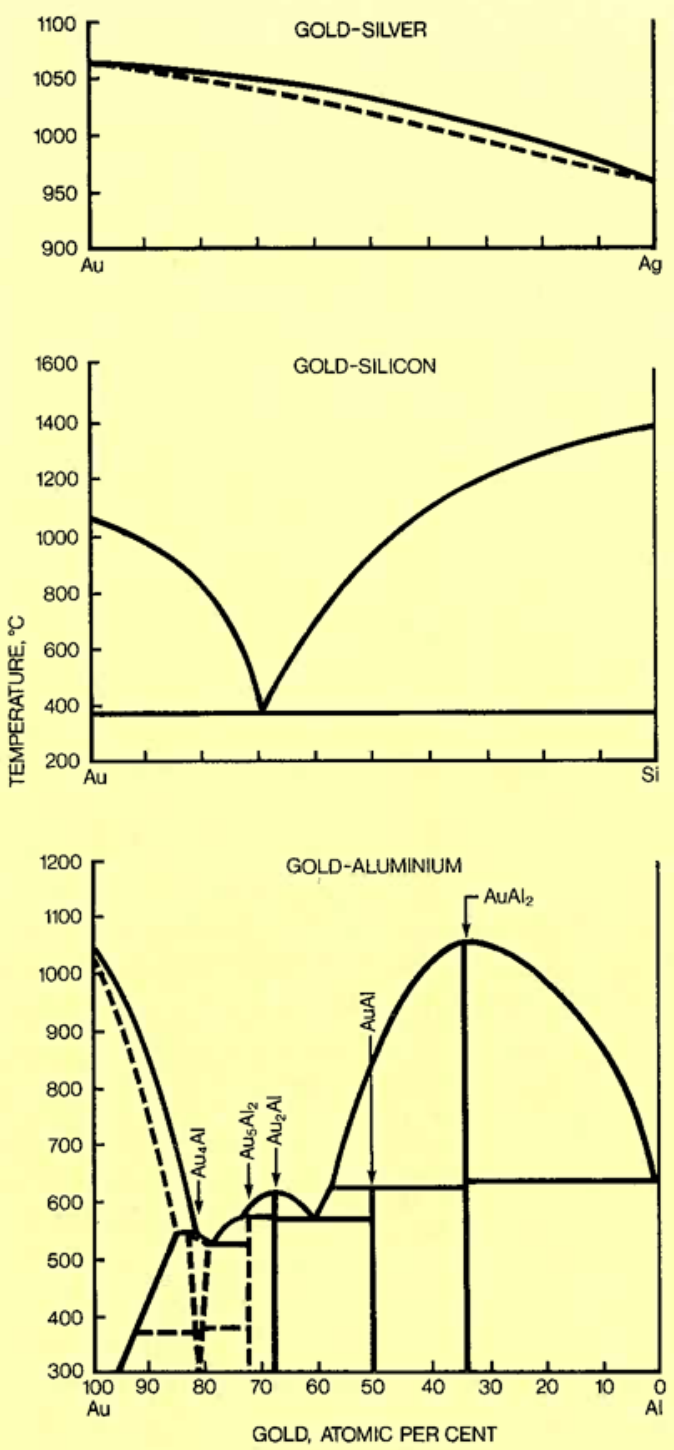

Fig. 3 Equilibrium phase diagrams for the goldsilver, gold-silicon and gold-aluminium systems tuitively explained by these diagrams. The reaction between solid gold and silicon at metallurgically low temperatures, for example, is probably a manifestation of the deep eutectic at $375^{\circ} \mathrm{C}$. Similarly, films of gold and aluminium react at low temperatures to form the intermetallic compound $\mathrm{AuAl}_{2}$. This compound is the notorious 'purple plague'. A most distressing symptom of its formation is loss of mechanical strength at gold-aluminium bonds. However, since the thin film reactions of primary interest are those that take place far below liquidus temperatures, the equilibrium phase diagrams may offer no more than clues to the phenomena that are likely to occur.

Two of the most significant features of reactions at metallurgically low temperatures are, first, the large scale diffusional mass transport that may be involved, and secondly, in cases of phase formation, their progression through well defined sequences. We can generally ascribe the large scale mass transport in thin films to the dominance of defect-enhanced or grain boundary diffusion. The reasons as to why phase formation follows certain sequences are less clearly understood. We can state, in quite general terms, that the phase having the lowest free energy is the one most likely to be formed, provided that there is no kinetic barrier to nucleation or growth. At present, however, the free energies of thin film systems are unknown. This is due to their inherent complexity, that is, to factors such as internal stress and defect structures. Despite such complexities, it will become apparent that in many cases a single process or parameter can dominate the reactions.

In this review, examples of diffusion or reactions between gold-based couples will be presented which illustrate the many features and variables which characterize thin film reactions in general. We cannot refer to all our work in this field, but will select the more pertinent cases. A more complete presentation is given in our recent book (5).

\section{Systems Forming Solid Solutions}

Before the advent of microscopic depth-profiling techniques it was difficult to formulate models of interdiffusion and reactions in thin film systems. It was generally recognized that grain boundary or defectenhanced diffusion is dominant at low temperatures and certain manifestations of such rapid diffusion had been observed. Measurements performed in recent years, using RBS or sputter-profiling, have further clarified this situation. Before discussing these, however, it seems useful to give an example of the experimental methods which are associated with the backscattering technique.

Figure 4 provides a schematic representation of the RBS spectra of thin film gold-silver couples before 
and after annealing. With the ion beam incident on the gold surface (Figure 4(a)), the as-deposited gold and silver films give separate peaks (these are shown in slightly simplified 'squared-off' fashion); after annealing the signals overlap in the region between the main peaks, thus revealing that interdiffusion has taken place. These scrambled spectra can be deconvoluted by computation to give the gold and silver components shown as the overlapping flanks in Figure 4(a). The actual concentration profiles are then calculated from these. By reversing the order of the films, that is with the beam incident on the lighter silver film (Figure 4(b)), and by the suitable choice of film thicknesses, the pure gold and silver peaks can be made to overlap, to form a summation peak. On annealing, gold diffuses to the free surface and silver towards the substrate, with both revealing their separate scattering profiles directly, as shown by the dashed line flanks in Figure 4(b).

In a previous article in this journal, Pinnel (1) gave a schematic representation of the grain structure of deposited films and showed that the diffusion of atoms along grain boundaries can be many orders of magnitude faster than lattice, or bulk, diffusion. We have recently performed experiments on gold-silver couples to demonstrate the difference between grain boundary and bulk diffusion (6). Self-supporting couples of silver, $100 \mathrm{~nm}$ thick, and gold, $80 \mathrm{~nm}$ thick, were prepared. One set of couples was polycrystalline, with an average grain size of $50 \mathrm{~nm}$, and the other set consisted of single crystals. Figure 5 shows the RBS spectra from these films before and after annealing at $150^{\circ} \mathrm{C}$. The film thicknesses were carefully chosen so that, with the beam incident on the silver side, the spectra in the as-deposited state overlapped completely.

It is evident that diffusion in the polycrystalline couples differs radically from that in the single crystal couples. For example, after annealing at $150^{\circ} \mathrm{C}$ for 40 minutes, interdiffusion is observed to the level of about 10 atomic per cent in a polycrystalline couple. However, no interdiffusion is discernible in a single-crystal couple after identical treatment (in Figure 5 , the points of the post-anneal spectrum lie exactly on the smooth line drawn through the preanneal spectrum). This finding is consistent with calculations based on the bulk or lattice diffusion coefficient for the gold-silver system, which predicts interdiffusion to a depth of only $10^{-4} \mathrm{~nm}$ after 40 minutes at $150^{\circ} \mathrm{C}$. With an experimental depth resolution of $15 \mathrm{~nm}$ in the present measurements, interdiffusion between the single crystals is observed only after anneals at 400 or $500^{\circ} \mathrm{C}$. The concentration profiles for single crystal gold and silver diffusing into each other have the classical shapes of the complementary error function curves (erfc) which are

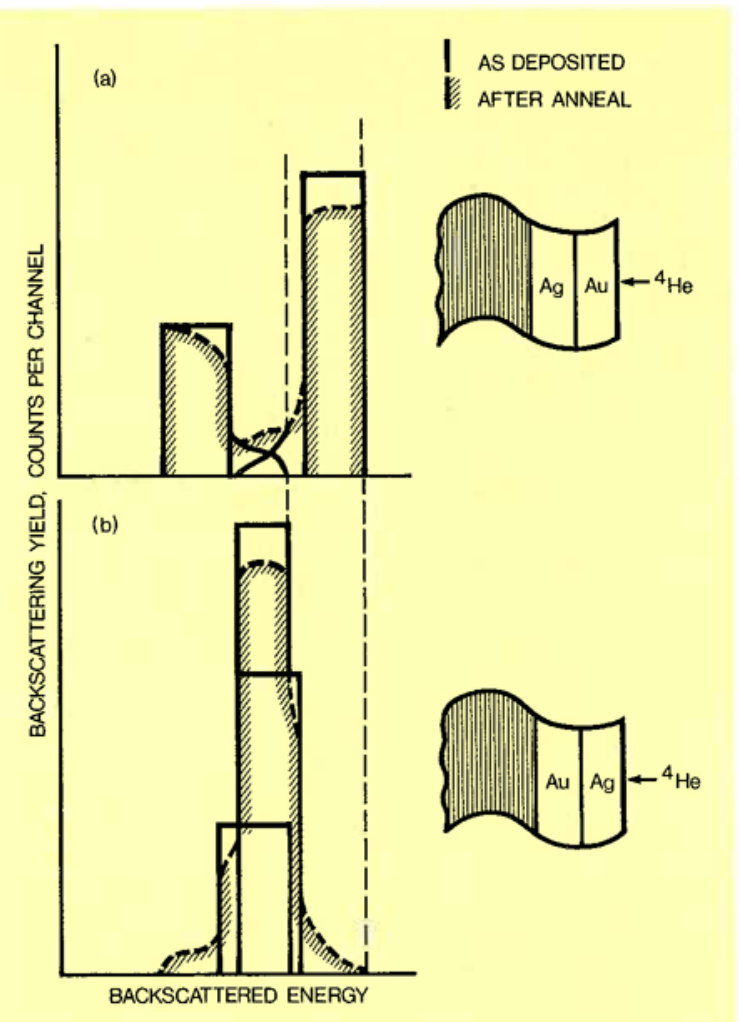

Fig. 4 Schematic representation of Rutherford backscattering spectra for interdiffusion between silver and gold. (a) With the gold/silver configuration, the peaks corresponding to the two metals are separate in the as-deposited condition and overlap after diffusion. (b) With the silver/gold configuration the peaks can be made to overlap in the as-deposited condition and, after annealing, interdiffusion is revealed in the spectrum by the dashed lines flanking the peak

predicted for lattice diffusion and from which lattice diffusion coefficients in agreement with published data can be calculated.

It is clear that diffusion in polycrystalline goldsilver couples is dominated by the short-circuiting action of defects. In fact, polycrystalline couples exhibit interdiffusion at room temperature, as shown by RBS spectra one week after evaporation (see the spectrum marked 'NO ANNEAL' in Figure 5). The question whether this truly represents a room temperature diffusion process, rather than one occurring during film deposition, is answered positively in Figure 6. This shows the concentration profiles of gold in a silver film immediately after deposition and after increasing periods of time at room temperature. The contention that gold is diffusing along silver grain boundaries is substantiated by the following: if the saturation value of 2 atomic per cent, shown in Figure 6, corresponds to saturation of the silver boundaries by gold, then simple geometric arguments (based on a grain boundary width of $0.5 \mathrm{~nm}$ ) require columnar grains $50 \mathrm{~nm}$ 


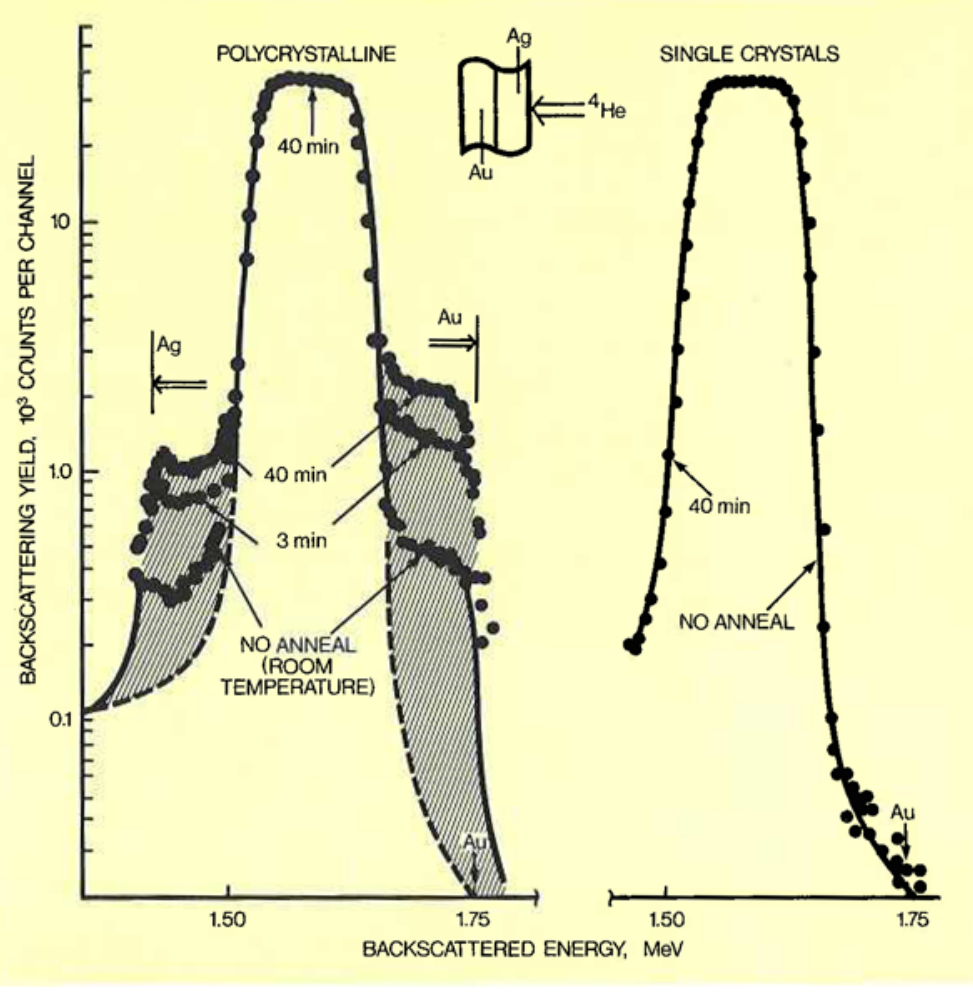

Fig. 5 Rutherford backscattering spectra from single erystal and polycrystalline gold-silver couples bombarded with 1.9 MeV helium-4 ions. Film thicknesses and the order of deposition were chosen to give overlap of the main gold and silver peaks in the asdeposited condition. The spectra before annealing are shown as solid lines. In the polycrystalline couple, annealing at $150^{\circ} \mathrm{C}$ results in the displacement of the gold peak towards the higher energy side and of that for silver towards the lower energy side. The positions of gold on the outside surface and silver on the substrate surface are indicated by open arrows. Note that in the single crystal couple interdiffusion is not detected after annealing for 40 minutes at $150^{\circ} \mathrm{C}$

in diameter. Transmission electron microscopy measurements indeed confirmed that the grains in our samples had a mean diameter of approximately this value. This experiment is an example of the need to combine measurements by several techniques in order to arrive at firm conclusions regarding the mechanism of interdiffusion.

By summing the number of gold atoms in the flat portion of the spectra (the 'WINDOW') in Figure 6, we can obtain the rate of interdiffusion as a function of temperature, as shown in Figure 7. The first point of interest is the initial dependence of room temperature diffusion on the square root of time. This corresponds to the diffusion of gold along silver grain boundaries. The later, lower rate corresponds to lattice diffusion. On heating above room temperature, however, there is initially a very rapid rise in the concentration of gold in silver, followed by much slower interdiffusion. As discussed earlier, the maximum concentration of gold arising from its presence in the silver grain boundaries only is of the order of 1 to 2 atomic per cent. Moreover, lattice diffusion from the grain boundaries into the interior of the grains can, at most, account for a few atomic per cent of gold under the experimental conditions discussed here. We believe that the high concentrations of gold result from the movement of grain boundaries during annealing and the consequent introduction of gold throughout the volume of the silver film. Once the network of grain boundaries has reached equilibrium, only normal lat- tice diffusion takes place and the gold concentration increases very slowly. Indeed, Cahn et al. (7) have recently shown that grain boundaries can migrate during diffusion, under a driving force supplied by the free energy of mixing or alloying. This free energy is generated by atoms which are deposited in the lattice in the wake of the migrating boundary.

It is important to obtain quantitative data on grain boundary diffusion coefficients, since the rapid interpenetration resulting from defect-enhanced diffusion is dominant in many surface and thin film phenomena. There are, at present, two general methods of determining such coefficients. First, is the Whipple analysis, which predicts that the concentration of the diffusing species is proportional to $\mathrm{X}^{6 / 5}$, where $\mathrm{X}$ is the distance from the interface. Figure 8 shows a Whipple plot of experimental data on palladium diffusion through gold films, obtained by Auger sputter profiling. Within $40 \mathrm{~nm}$ of the interface erfc diffusion predominates, as predicted by the Whipple model. (The apparent linearity of $\ln \left(\operatorname{erfc}\left(\right.\right.$ concentration)) with $\mathrm{X}^{6 / 5}$ is fortuitous in this range of concentration.) The presence of grain boundary diffusion is shown by the breaks in the curves and by the linear regions beyond $40 \mathrm{~nm}$. It is from the slopes of these lines that the grain boundary diffusion coefficients are calculated. The most accurate values for these coefficients are obtained when the grain boundaries are well separated, and this unfortunately occurs mainly in thick films rather than in the thin 


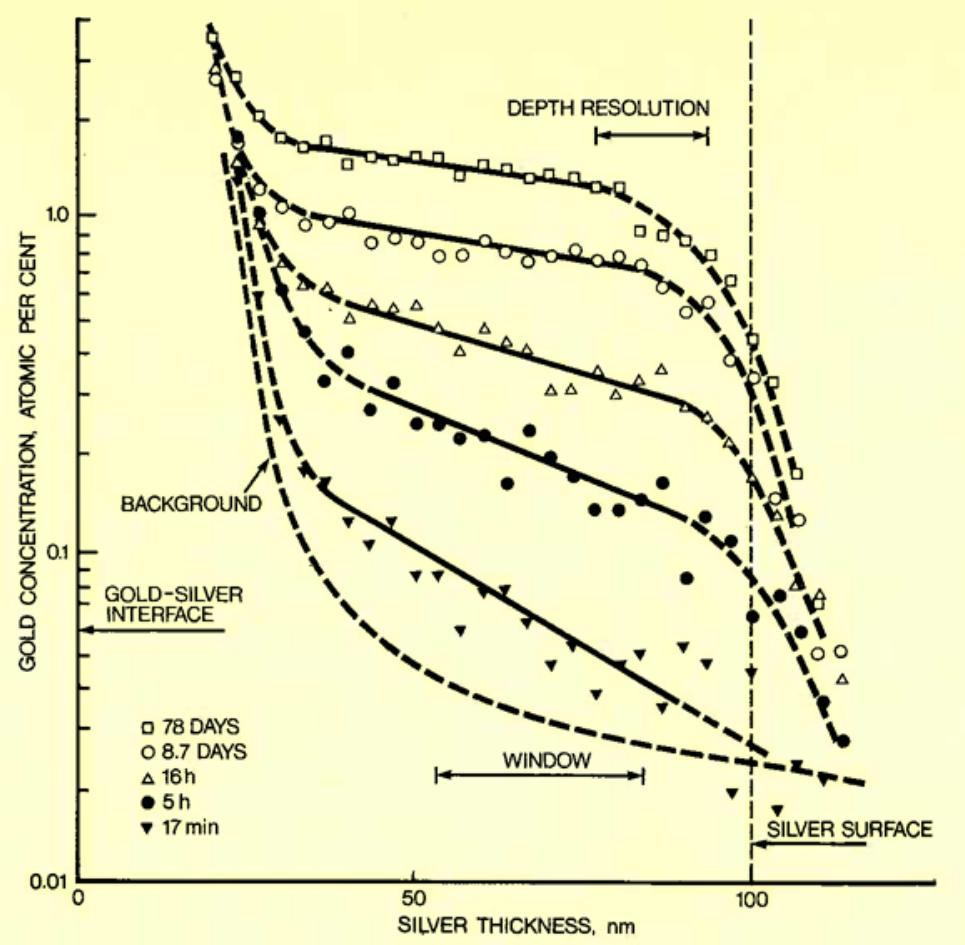

Fig. 6 Gold concentration profiles after its diffusion for various periods of time at room temperature in a polycrystalline silver film. The apparent location of some experimental points in the region 'outside' the free surface of the silver film is due to the limitations of depth resolution of the Rutherford backscattering technique

films which are of primary practical interest. A second, indirect method of determining grain boundary diffusion coefficients consists of measurements of the time dependence of the build-up of substrate material at the surface of the deposited films (9).

We are now in the position to estimate the rate of grain boundary diffusion in gold films with some confidence (10). However, because of the complexities introduced by the motion of grain boundaries in solid solution systems, it is still quite difficult to arrive at accurate predictions of the quantities of interdiffused materials.

\section{Oxidation, Chemical and Barrier Effects}

The experiments described in the previous section were carried out under clean, vacuum conditions. The device engineer is concerned with the behaviour of thin films in more hostile environments, where the influence of oxidizing or corrosive contaminants can be quite complex. We will illustrate some of these effects by reference to the gold/palladium/titanium system (11). Figures 9 and 10 show the effects of annealing gold/titanium and palladium/titanium couples in air and in vacuum. Although the interdiffusion of titanium and gold is faster than that of

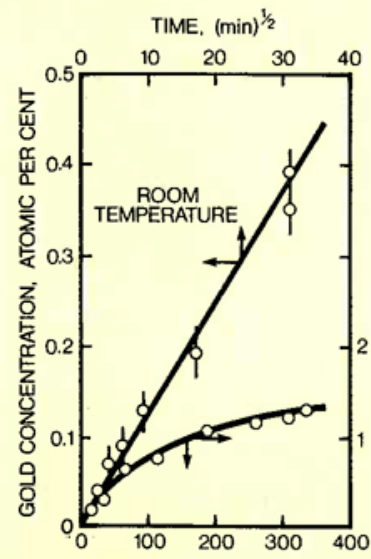

TIME, $(\min )^{1 / 2}$

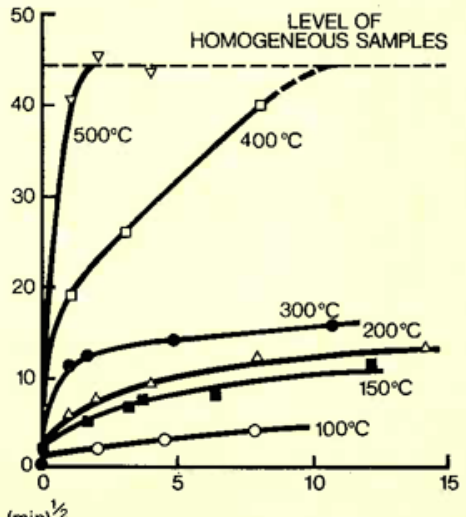

Fig. 7 Mean concentration against time of gold diffusing into a polycrystalline silver film at various temperatures. At room temperature (left), the initial proportionality of concentration to the square root of time indicates grain boundary diffusion; the later, slower rise in gold concentration corresponds to lattice diffusion. At elevated temperatures (right), the initial very rapid rise of gold concentration might well be caused by the displacement of gold-containing silver grain boundaries; the subsequent, slower diffusion rate again corresponds to lattice diffusion once the network of goldsaturated silver grain boundaries has stabilized 


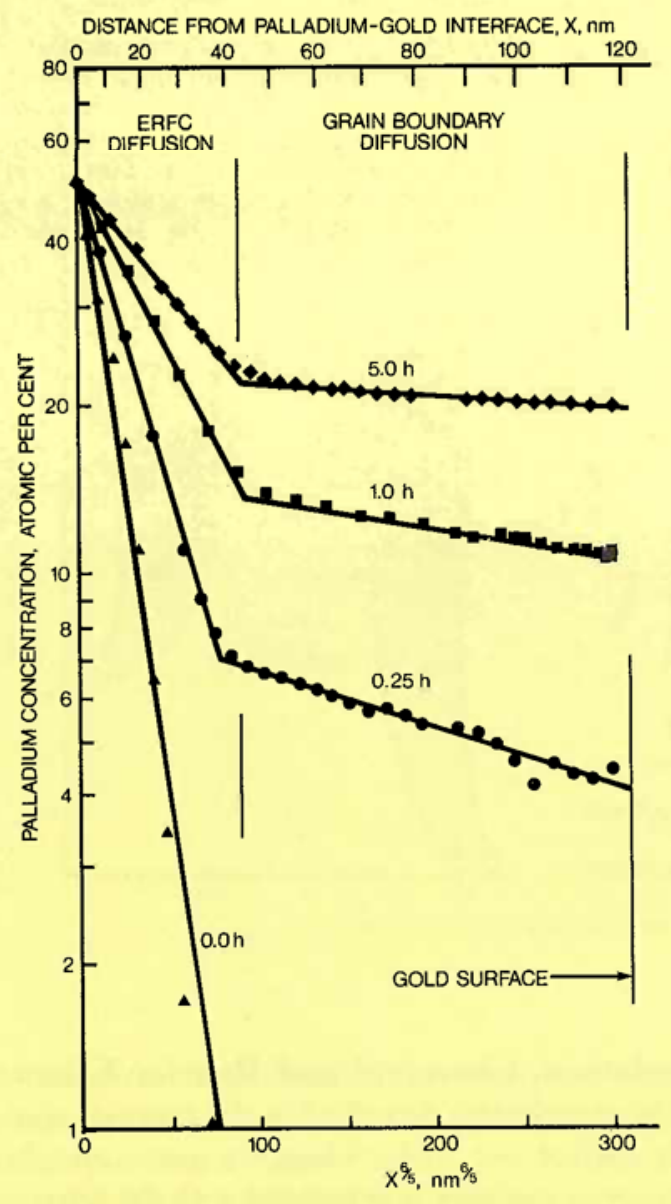

Fig. 8 Whipple plot for palladium diffusing into a gold film at $250^{\circ} \mathrm{C}$. Grain boundary diffusion coefficients are calculated from the slopes of the lines in the region beyond $40 \mathrm{~nm}$

titanium and palladium, the general effects of vacuum annealing are similar in both systems. When the couples are annealed in air, however, their behaviour is markedly different. In the case of the gold/titanium system, titanium diffuses along the grain boundaries to the free surface of the gold where it is oxidized. The surface oxidation of the titanium, by creating a chemical potential sink, continually enhances the interdiffusion. In the case of air-annealed palladium/titanium couples, however, very little interdiffusion is observed. The following competing processes occur simultaneously during annealing under oxidizing conditions: (1) interdiffusion of the titanium and palladium, (2) diffusion of oxygen through the palladium, and (3) oxidation of titanium near the palladium-titanium interface. As soon as the latter process reaches a critical stage, most likely when a continuous titanium oxide layer is formed along the palladium grain boundaries in the vicinity of the interface, further diffusion of titanium in palladium is blocked. Therefore, oxygen enhances diffusion in the gold-titanium system, but ultimately blocks diffusion in the palladium-titanium system.

The very rapid diffusion of titanium through gold films and the formation of titanium oxide at the free surface explains some of the problems experienced with gold/titanium metallizations. What is the role of an intermediate barrier metallization of palladium? Figure 11 shows the RBS spectra of a gold/palladium/ titanium metallization before and after annealing, where the dashed lines are the deconvoluted concentration profiles. The same diffusion patterns are observed irrespective of whether annealing is carried out in air or in vacuum. It is remarkable that no diffusion of titanium is evident in this three-layer system, despite the copious diffusion observed in isolated palladium-

Fig. 9 Rutherford backscattering spectra for gold/titanium thin film couples annealed in air and in vacuum. Note the diffusion of titanium to the surface of the gold 
Fig. 10 Rutherford backscattering spectra for palladium/titanium thin film couples annealed in air and in vacuum. Note the absence of interdiffusion on air annealing

titanium couples. We believe that this situation arises because the grain boundary diffusion of gold in palladium is so rapid that the palladium grain boundaries are effectively blocked by gold.

Clearly, the ability to establish diffusion barriers and to maintain the electrical integrity of metallizations is of crucial importance in many applications of thin films. The role of the palladium in the foregoing discussion is complex and not immediately obvious. One important consideration is that in nearly all metallic systems, grain boundary diffusion constitutes the dominant diffusion mechanism. If we could fabricate single crystal barrier layers, interdiffusion would be minimized. Such an approach does not, however, appear practical. An alternative, which is used in many applications, is to make the barrier of a stable intermediate phase. Thus, in some gold-based systems, titanium nitride is introduced as an additional barrier (12).

\section{Layered Compounds}

In the preceding discussion we have concentrated on metallurgical systems which do not form well defined phases, but rather exhibit extended solubility. Many metal combinations, however, react to form stable phases having structures and chemical bonding which differ from those of the starting materials. An example is the gold-aluminium system, the binary phase diagram of which shows many stable intermediate phases (Figure 3(c)). This system has attracted much interest from thin film experts because of its obvious technological importance and also because it serves as an ideal model.

It would be advantageous to study the growth of a compound phase between two films, all in single crystal form, as was done in the case of the simpler solid solution system of gold and silver. Such a system would be convenient to establish the limiting conditions of reaction. This, however, would require the structures of the compound and of the materials of the starting couple to be the same. Unfortunately no such system is known.

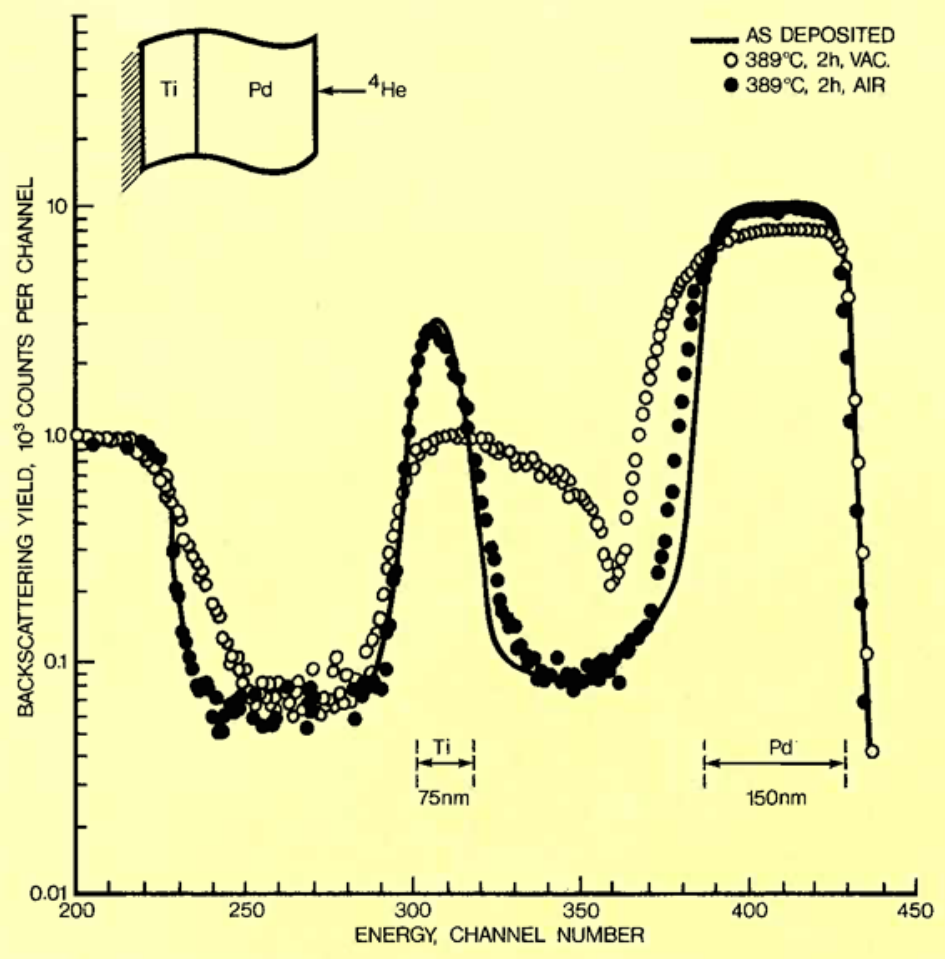

Fig. 11 Rutherford backscattering spectra for a gold/palladium/titanium metallization before and after vacuum annealing. Note the interdiffusion between palladium and gold, but the absence of diffusion of titanium in the palladium 


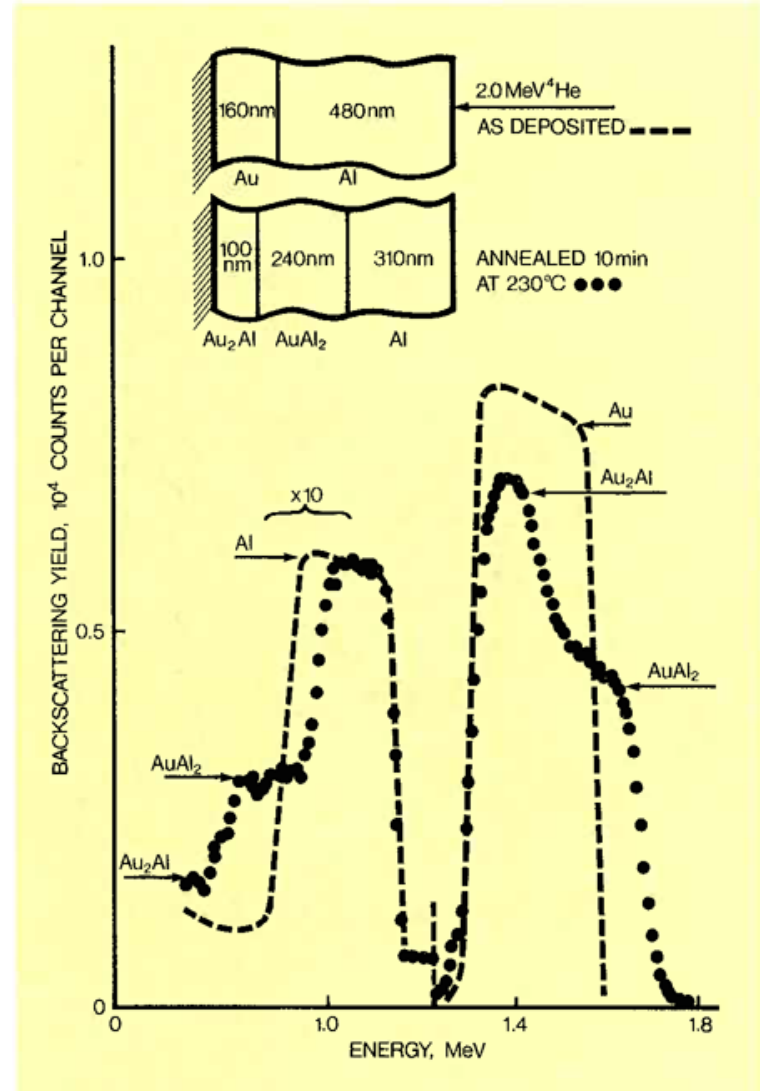

Fig. 12 Rutherford backscattering spectra for the aluminium/gold thin film system. Annealing at $230^{\circ} \mathrm{C}$ results in the formation of the intermetallic compound $\mathrm{Au}_{2} \mathrm{Al}$ and, at the interface between it and aluminium, of the further compound $\mathrm{AuAl}_{2}$. The arrows indicate calculated spectrum peak heights for $\mathrm{Au}_{2} \mathrm{Al}$ and $\mathrm{AuAl}_{2}$. After (13)

What is observed in many metal-metal and metalsemiconductor reactions is that the compound phase grows in a well defined layered structure at the interface (Figure 1). Metal silicide contacts in fact enjoy such widespread use because of their remarkable lateral uniformity.

The gold-aluminium system similarly features the formation of laterally uniform phases. Figure 12 shows the formation of intermediate layers of $\mathrm{Au}_{2} \mathrm{Al}$ and $\mathrm{AuAl}_{2}$. The phase diagram (Figure $3(\mathrm{c})$ ) indicates the stabilities of $\mathrm{Au}_{4} \mathrm{Al}, \mathrm{Au}_{5} \mathrm{Al}_{2}, \mathrm{Au}_{2} \mathrm{Al}, \mathrm{AuAl}$ and $\mathrm{AuAl}_{2}$ at temperatures below $250^{\circ} \mathrm{C}$. The presence of all, except AuAl, has been detected in the past during measurements on gold-aluminium diffusion couples. However, not all the phases form at one time and, in fact, the entire sequence of reactions and the final phase structure depend systematically on the temperature of the reaction and on the atomic quantities of gold and aluminium involved. Campisano et al. (13) and Majni et al. (14) have carefully followed the growth sequence of the phases. Their results are shown schematically in Figure 13. The composition of the final phase is determined by the proportions of the diffusing species. By carefully depositing equal thicknesses of aluminium and gold it has recently been possible to form the elusive AuAl compound during diffusion.

While the sequence of phase formation in the goldaluminium system is now well understood, the corresponding reaction mechanisms and the respective roles of grain boundary and bulk diffusion remain unknown. We (15) have compared the diffusion in a polycrystalline gold-aluminium couple with that occuring in a system consisting of a single crystal gold film, $120 \mathrm{~nm}$ in thickness, overlaid by a polycrystalline film of aluminium, $400 \mathrm{~nm}$ in thickness. The rate of diffusion in the single crystal configuration was at least one order of magnitude lower than that in the polycrystalline configuration. This result would indicate that grain boundary diffusion is the dominant mechanism of transport in systems forming compound phases.

\section{Reactions with Semiconductors}

Gold reacts with silicon and with compound semiconductors at temperatures that are very low in metallurgical terms. This behaviour, while troublesome in many cases, can be used to advantage. For example, gold reacts with gallium arsenide at temperatures of approximately $200^{\circ} \mathrm{C}$ as revealed by the outdiffusion of gallium through gold films on the semiconductor (16). The resulting interface favours the formation of ohmic contacts. In industrial applications, most ohmic contacts to gallium arsenide are, in fact, formed using thin films of gold-germanium or gold-nickel-germanium alloys.

Gold is not employed in ohmic contacts on the active areas of silicon chips, since melting results in 'balling-up' (decomposition of the continuous gold film into discrete particles of eutectic) and the reaction is not easily controlled. Gold contacts are, however, applied to the back of wafers, where precise control of lateral morphology is less important. Moreover, the gold-silicon eutectic can be utilized in the bonding of silicon device chips to gold-coated ceramic packages.

It is intriguing to consider why gold reacts with semiconductors in the solid state at temperatures well below the eutectic point. While it is known that many low temperature reactions between metals in contact with one another are due to defect-enhanced or grain boundary diffusion, this is clearly not the case where metals are in contact with single crystal semiconductors. One explanation that has been suggested (17) for such reactivity is based upon the observation that the metals that react with silicon at low temperature also diffuse interstitially within it. At the metal-silicon interface, a metal atom is pictured as transferring into 
an interstitial site, thus weakening the bonding in the silicon. Such weakening, which can be visualized as a transformation from covalent to metallic bonding, could account for metal-semiconductor reactions at temperatures as low as $200^{\circ} \mathrm{C}$.

\section{Conclusions}

In this review we have discussed fundamental studies on interdiffusion and on the chemical reactions that occur between gold films and various metals and semiconductors at metallurgically low temperatures. These studies have lead to a much improved understanding of thin film behaviour. The practical importance of gold films in the electronics industry has been the chief driving force in this work. With the increasing demand for reliability and for further miniaturization of electronic devices, an even deeper understanding of reactions in thin films will be required.

\section{References}

1 M. R. Pinnel, Gold Bull., 1979, 12, (2) 62-71

2 P. H. Holloway, Gold Bull., 1979, 12, (3), 99-106

3 J. W. M. DuMond and J. P. Youtz, Phys. Rev., 1935, 48, 703

4 J. W. M. DuMond and J. P. Youtz, f. Appl. Phys., 1940, 11, 357-365

5 'Thin Films - Interdiffusion and Reactions', edited by J. $M$. Poate, K. M. Tu and J. W. Mayer, John Wiley, New York, 1978

6 R. G. Kirsch, J. M. Poate and M. Eibschutz, Appl. Phys. Lett., 1976, 29, 772-775

7 J. W. Cahn, J. D. Pan and R. W. Balluffi, Scr. Metall., 1979 13, 503-509

8 P. M. Hall, J. M. Morabito and J. M. Poate, Thin Solid Films, $1976, \mathbf{3 3}, 107-134$

9 J. C. M. Hwang and R. W. Balluffi, F. Appl. Phys., 1979, 50, 1339-1348

10 D. Gupta, D. R. Campbell and P. S. Ho; see (5), Chapter 7

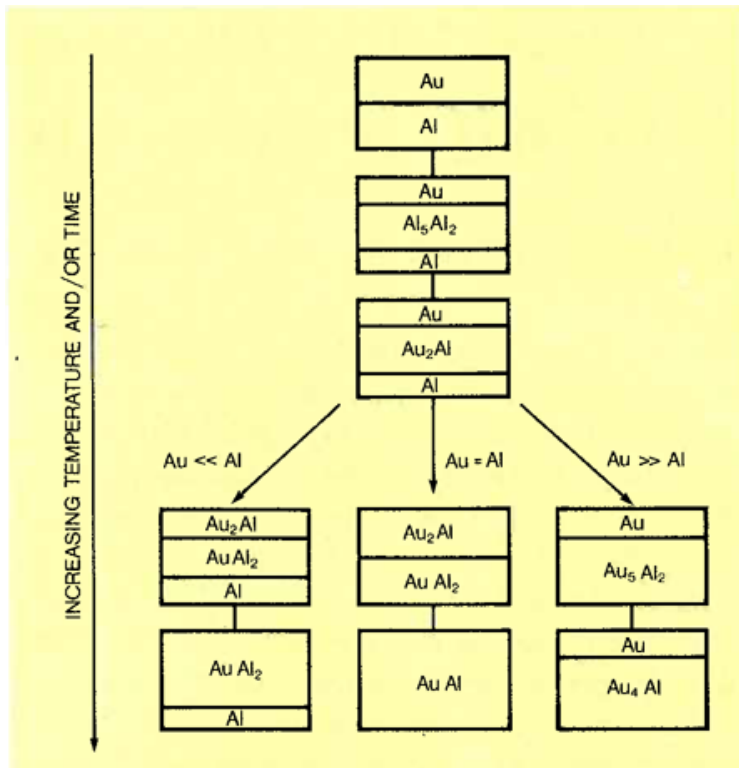

Fig. 13 Schematic representation of compound formation in gold-aluminium thin film systems. The stoichiometry of the final compounds is determined by the annealing temperature and by the proportions of the starting materials. After (14)

11 J. M. Poate, P. A. Turner, W. J. DeBonte and J. Yahalom, 7. Appl. Phys., 1975, 46, 4275-4283

12 M. A. Nicolet, Thin Solid Films, 1978, 52, 415-443

13 S. U. Campisano, G. Foti, E. Rimini, S. S. Lau and J. W. Mayer, Philos. Mag., 1975, 31, 903-917

14 G. Majnı, G. Ottaviani and E. Galli, 7. Cryst. Growth, 1979, 47, (4), 583-588

15 R. G. Kirsch, J M. Poate and M. Eibschutz, unpublished work

16 A. K. Sinha and J. M. Poate; see (5), Chapter 11

17 K. N. Tu, Appl. Phys. Lett., 1975, 27, 221-224 